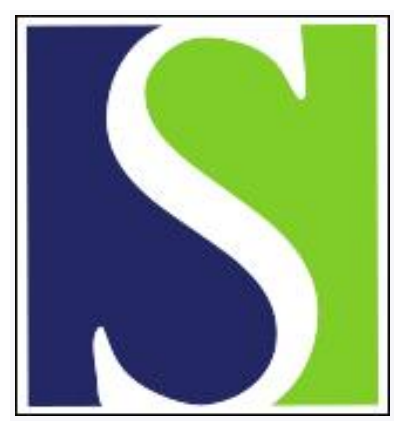

Scand J Work Environ Health 1989;15(1):13-17

https://doi.org/10.5271/sjweh.1889

Issue date: Feb 1989

Carcinogenesis and teratogenesis may have common mechanisms.

by Vainio $\mathrm{H}$

Affiliation: Department of Industrial Hygiene and Toxicology, Institute of Occupational Health, Helsinki, Finland.

This article in PubMed: www.ncbi.nlm.nih.gov/pubmed/2537999

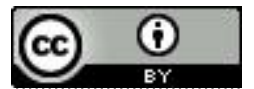




\title{
Carcinogenesis and teratogenesis may have common mechanisms
}

\author{
by Harri Vainio, $\mathrm{MD}^{1}$
}

\begin{abstract}
VAINIO H. Carcinogenesis and teratogenesis may have common mechanisms. Scand $J$ Work Environ Health 1989;15:13 - 17. The specific mechanisms of carcinogenesis and teratogenesis are poorly understood. There are, however, some known or potential common mechanisms, such as gene or chromosome mutations, interference with gene expression, altered membrane properties, or altered intracellular homeostasis. Carcinogenesis is generally regarded as a multistage process, and a carcinogen can act at one or several stages. Agents acting in the early stages of the neoplastic process are DNA-reactive, mutagenic compounds which enable cells to be transformed to malignancy. These agents can also, if acting during critical periods of ontogenesis, induce abnormal development of the embryo. Agents which block gap junctional intercellular communication may act both as tumor-promoting agents and as teratogens in the developing embryo. Hormones are essential in the control of development and differentiation. Modulation of the intracellular hormone receptors may lead to changes in homeostasis with abnormal cellular proliferation and development as a consequence.
\end{abstract}

Key terms: hormone receptors, intercellular communication, mutagenesis, tumor promotion.

Most forms of cancer are brought about by a multistage process involving initiation, promotion, and progression. Most so-called "complete" carcinogens act as initiators bringing about the first irreversible step in carcinogenesis. This complex process involves several mechanisms, some of which are still not well understood $(1,2)$.

Without it being suggested that teratogenesis is the result of a single or simple errant mechanism, there is little evidence that teratogenesis involves the same multistage mechanistic processes that occur in carcinogenesis. Nevertheless, the initiation and promotion stages of carcinogenesis may have the same molecular bases as teratogenesis (3). The fact that gene regulation is crucial for the control of cell differentiation and development is generally accepted. It is known that interference in the process of normal differentiation and development can lead to abnormal growth, malformation, and neoplasm. Thus, at the level of cellular differentiation, carcinogenesis and teratogenesis may be biologically linked.

\section{Carcinogenesis as a complex biological phenomenon}

A carcinogen may act during one or several of the stages in the neoplastic process. Especially pertinent to cancer is the activation, by carcinogens, of a certain class of genes, called oncogenes, that appear to

\footnotetext{
1 Department of Industrial Hygiene and Toxicology, Insti-
} tute of Occupational Health, Helsinki, Finland.

Reprint requests to: Professor H Vainio, Institute of Occupational Health, Topeliuksenkatu 41 a A, SF-00250 Helsinki, Finland. control cellular growth. Mutation or gross deoxyribonucleic acid (DNA) rearrangement of these genes, such as chromosomal translocation or gene amplification, may result in the loss of their capacity to control cell replication. The conversion of oncogenes, which is triggered by a variety of factors ("carcinogens"), could be the final common step in the biological mechanism of carcinogenesis.

Activation of an oncogene (such as a ras protooncogene) is probably an early event in tumorigenesis and may even be the "initiation" event in some cases. Oncogenes can be roughly classified into two groups - those functioning in the nuclei and those functioning in the cytoplasm, including the cell membrane (4). And it may be that one of each group must be activated for carcinogenesis to occur. Some of the proteins that are encoded by oncogenes are located in nuclei (eg, those encoded by myc oncogenes), and others are located outside the nuclei (eg, those encoded by ras oncogenes) (5). P21 protein, which is encoded by cellular ras genes, is the best known oncogenic gene product. Normal P21 has both guanosine 5'-triphosphate-binding (GTP-binding) and GTPase activity, but carcinogen-activated P21 has greatly reduced GTPase activity. Amino acid substitutions at positions 12 and 61 reduce GTPase activity but have no effect on GTPbinding. The significance of GTPase activity lies in the analogy with the "G proteins" which, like P21, have GTP-binding capacity and can hydrolyse GTP to guanosine 5'-diphosphate (GDP) but, in addition, are known to function as intracellular transducers of growth regulatory signals from cell surface receptors.

Once cells in certain parts of the body are released from normal growth control, they begin to proliferate, overwhelm normal cells, and invade and distort 
normal tissue. Evidence obtained from several studies on experimental animals suggests that activation of ras protooncogenes is an early event in this process, although it is not sufficient to give full tumorigenic properties to the cells which harbor them. Activated ras genes have been detected in many benign (in situ) tumors, including mouse skin papillomas and liver adenomas, and in a large variety of human tumors (6). On the average, however, activated oncogenes have been detected in only $15-20 \%$ of human malignancies.

Yamasaki et al (7) have shown that the c-Ha-ras oncogene can be activated transplacentally in mice through a specific point mutation caused by a chemical carcinogen [7,12-dimethylbenz[a]anthracene (DMBA)], but the cell containing this mutation may remain dormant until it encounters (postnatally) a tumor-promoting stimulus. Thus, a single transplacental dose of DMBA can induce a mutation (A to T transversion) at the 61 st codon of the cellular Ha-ras gene, which remains dormant until the cells are promoted (eg, by skin painting with a phorbol ester). Cells "initiated" in this way can remain silent for a long time, and therefore a long-term memory of initiation on mouse skin must exist.

The identification and molecular characterization of human oncogenes have validated the old hypothesis that cancer is a genetic disease that is initiated by the occurrence of somatic mutations. However, it has also become evident that cancer development is a multistep process requiring the activation of many growth-controlling genes. Somatic hybridization of normal cells with malignant ones shows that the normal genome may enable tumor cells to respond to appropriate growth-controlling stimuli in vivo. Genes that can inhibit expression of the tumorigenic phenotype have been called "tumor suppressor genes" or "antioncogenes"' $(8,9)$.

\section{Biological basis for abnormal embryogenesis}

The term "reproductive toxicant" covers a mixed group of agents which interfere with the ability of males and females to reproduce. A "developmental toxicant" can produce adverse effects on the developing conceptus after exposure during pregnancy. These effects may be manifested in the embryonic or fetal periods or postnatally.

One manifestation of developmental toxicity is structural malformations in the conceptus as a consequence of exposure of the mother during pregnancy. Teratogenesis is thus a process of abnormal embryonic development. The definition of teratogenesis is often restricted to structural abnormalities; thus nonstructural deficits such as behavioral deficits, mental and physical growth retardation, immunological compromise, and increased susceptibility to carcinogenesis are, inappropriately, excluded. Spontaneous abortions, premature delivery, and stillbirth are additional manifestations of embryotoxicity which may share common mechanisms with teratogenesis.

The processes of teratogenesis have been discussed by Wilson (10), who outlined the early events that could contribute to abnormal embryonic development and teratogenesis. Mutation is probably the most firmly established mechanism of teratogenesis. It has been estimated that $20-30 \%$ of human developmental errors can be attributed to mutation in a prior germ line. When somatic mutations occur in an early embryo, a demonstrable structural or functional defect may be produced. Other pivotal effects include cell death and altered membrane characteristics and the processes that depend on them, such as tissue induction, cell recognition, responsiveness to hormonal stimuli, and formation of intercellular junctions.

Intercellular communication has been an integral consideration in most theories of embryogenesis. It has been reasoned that the cells of an embryo must be able to communicate with each other in order to define tissue specificity and pattern formation and to coordinate morphogenetic events. In addition neighboring tissues have long been known to exert inductive influences on each other and therefore require some sort of transmission of signals.

\section{Carcinogenesis and teratogenesis may have common mechanisms}

Associations between teratogenesis and carcinogenesis have been observed at several levels. Because of the complexity of each stage, many mechanisms are probably involved. Some of the biological steps of potential importance in carcinogenesis, as well as in teratogenesis, are shown in figure 1.

\section{Tumor initiation, mutagenesis, and teratogenesis}

DNA-reactive alkylating agents are usually also carcinogens. As chemical mutagens, they can induce permanent alterations of genetic information and can act both as initiators and as "complete" carcinogens. However, genotoxic agents are not necessarily mutagenic in normal in vitro assays. Reynolds et al (11) found that furan and furfural were not mutagenic in the Ames Salmonella assay (and would be classified as "nongenotoxic" on this basis), but that they caused liver tumors activating mutations in several codons of the ras gene of mice. Thus, although furan and furfural were not active in tests designed to test for mutagenicity, the authors considered that the mutations in tumor oncogenes were evidence that they are mutagenic. Agents that do not damage DNA can also cause karyotypic changes, which can result in chromosome mutations (polyploidy, aneuploidy, nondisjunction) that may lead to a deregulation of cellular growth.

Cancer initiation is an irreversible event involving mutagenesis, and mutagenesis in the germ line can contribute to genetic diseases. Furthermore, mutagenesis 
in somatic cells plays a role not only in carcinogenesis but, when occurring in the early embryo, also in teratogenesis (10).

\section{Tumor promotion and teratogenesis}

Some chemicals (known as tumor promotors) which have little or no genotoxic activity can drastically enhance the incidence of tumors, eg, on the backs of mice that have been treated previously (initiated) with an alkylating agent. (Such as DMBA, see reference 7.) Tumor promotion has been observed in many tissues in numerous animal model systems with high tissue and species specificity. However, very little is known about the mechanisms of tumor promotion.

One popular theory is based on the effects of promoters on intercellular communication. When mammalian cells come into contact with one another, small membrane channels, known as gap junctions, may form between them. These gap junctions permit the transfer of small molecules that may regulate cell proliferation and differentiation (12). Tumor promoters can interrupt intercellular communication between a variety of cell types, and this effect may have a mechanistic relationship with promotion.

Cells within a tissue communicate with each other not only by secreting molecules into the extracellular space, but also by passing signals directly through intercellular channels. Mechanistic studies suggest that cells exchange low-molecular-weight components $(<2000 \mathrm{D})$ via gap junctions and that this activity is regulated by phosphorylation dependent on adenosine 3,'5'-cyclic monophosphate (cAMP) (13). These intercellular signals presumably participate in the regulation of growth and differentiation so that their disturbance could represent a step in the process of carcinogenesis or teratogenesis.

Initiation of the carcinogenic process may begin with the production of a single tumor cell. This cell is probably in contact with a number of normal cells and would thus receive growth regulatory substances from them via gap junctions. As a result, the phenotypic expression of malignancy in the tumor cell would be suppressed. Tumor promoters might act by a disrupting gap junctional function, preventing phenotypic suppression and allowing the tumor cell to proliferate. Numerous types of evidence support this hypothesis, such as the observation that many tumor promoters inhibit intercellular communication (14).

Gap junctional communication also plays a very important role in the adaptation of multicellular organisms, as well as in various developmental processes, morphogenetic field determination, and tissue induction (15). Several teratogens that are not known to be alkylating or DNA-reactive agents, including mirex, various alkyl glycol ethers, chlorpromazine, and ethanol, can block intercellular communication. Direct interruption of junctional communication may thus be a mechanism of action for teratogens. At the cellular membrane, these substances may also activate cAMP reactions by binding to their receptors. Since increased intracellular levels of cAMP and cAMP-dependent protein kinase activity have been associated with recovery of junctional competence, it is possible that substances that activate the CAMP cascade may also influence junctional communication. Gap junctional changes have been associated with cAMP-dependent hormones in several biological systems.

\section{Modulation of hormone receptors}

Hormones are essential for controlling normal development and differentiation; for instance, estrogen is necessary for normal reproduction and the maintenance of pregnancy and, in both males and females, affects other hormones that regulate homeostasis and reproductive function. In cells, estrogen binds to and activates a cytoplasmic receptor, and this estrogen-estrogen receptor complex is translocated into the nucleus, where it acts like a transcriptional regulator (16).

2,3,7,8-Tetrachlorodibenzo-para-dioxin (TCDD) is a highly toxic environmental pollutant that can cause cancer in rodents; it is also teratogenic in rodents. It is not genotoxic, ie, not mutagenic, and it does not react with DNA. A striking feature of the toxicity of TCDD is its ability to deplete (atrophy) lymphoid tissues in general and thymus in particular, this deple-

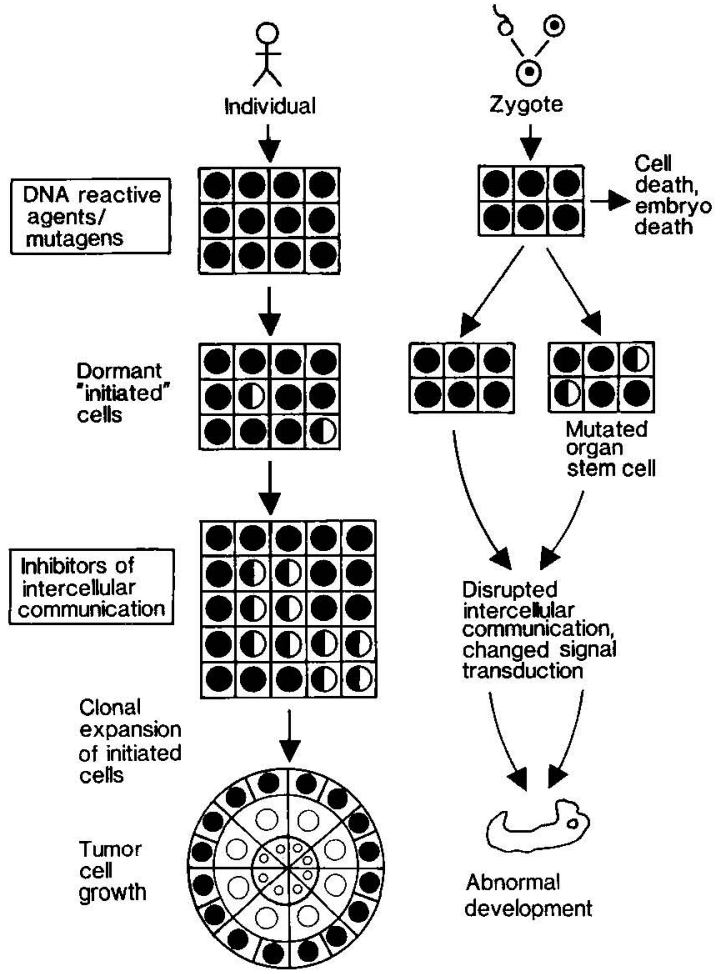

Figure 1. Schematic representation of mechanisms important in the processes of carcinogenesis and teratogenesis. 
tion being manifested clinically as immune deficiency. (For a review, see reference 17.)

TCDD has been shown to interact with specific, soluble binding sites in target tissues. The large interand intraspecies differences in both acute toxicity and teratogenesis can be attributed to events that occur after receptor binding (18). A major determining factor in TCDD toxicity seems to be the species- and tissuespecific control over a battery of enzymes, which are expressed or repressed after TCDD binds to its receptor. Recently, it has been suggested that the biological effects of TCDD are based on a modulation of the estrogen receptor (19). It has also been suggested that the resistance of some animal species (eg, hamster) to the toxicity of TCDD is due to species' ability to synthesize estrogen and thus increase its tissue concentrations during exposure.

Exogenous estrogens are also immunosuppressive and cause thymic involution. Other hormones and growth factors can modulate the effects of estrogen. For example, some breast cancers appear to be estrogen-dependent and can be arrested by antiestrogens $(20,21)$.

\section{Concluding remarks}

Many carcinogens are also teratogens, but many teratogens are not carcinogens. Carcinogenesis is a multistage process, and a carcinogen can be active at one or more of these stages. The simplest operational model distinguishes initiation from promotion. Initiators induce genotypic changes, and promotion results in clonal expansion of the initiated cells. Initiating activity reflects the capacity of an agent to produce irreversible cellular changes and gives cells the potential to progress to tumors. This phenomenon involves changes in the growth-related genes (oncogenes). While a genetic change in the target cell is likely to represent an early required event in malignant transformation, other differentiation-related events may be important for tumor promotion.

In a given tissue, both in the developing embryo and in stationary tissue, cells are maintained in homeostasis by various forms of intercellular communication, gap junctions being the factor believed to play the most important role. Disturbance of this orderly, structured cell society may modulate cell proliferation and differentiation and lead either to the process of teratogenesis or to the process of malignant growth. Many of the tumor promotors that have been shown to be teratogenic inhibit gap junctional communication. Modulation of intracellular receptor proteins can lead to drastic changes in cellular homeostasis, which, under certain circumstances, can also lead to reproductive failure, abnormal growth, and cancer. These modulators need not be DNA-reactive substances, but they must be able to interact with receptors. It has been suggested that the effects of some nongenotoxic agents, such as TCDD, are mediated by the modulation of es- trogen-binding receptors and lead to both teratogenesis and carcinogenesis.

Thus agents that act at the genetic level as initiating agents, as well as agents with tumor-promoting activity, may also have teratogenic effects if the developing embryo is exposed at a critical moment. The mechanisms are still largely unknown, but recent advances in molecular biology have provided new tools for this research.

\section{Acknowledgments}

I wish to thank Ms E Heseltine for her editorial assistance and Ms R Marttala for her secretarial help.

\section{References}

1. Montesano R, Bartsch H, Vainio H, Wilbourn J, Yamasaki $H$, ed. Long-term and short-term assays for carcinogenesis: a critical appraisal. I yon: International Agency for Research on Cancer, 1986. (IARC scientific publications no 83 .)

2. Cerutti PA. Response modification creates promotability in multistage carcinogenesis. Carcinogenesis 1988;9: $519-26$.

3. Trosko JE, Chang CC. Implications for risk assessment of genotoxic and non-genotoxic mechanisms in carcinogenesis. In: Vouk VB, Butler GC, Hoel DG, Peakall DB, ed. Methods for estimating risk of chemical injury: human and non-human biota and ecosystems. Chichester (England): John Wiley \& Sons, 1985:181-200.

4. Weinberg RA. The action of oncogenes in the cytoplasm and nucleus. Science 1985;230:770-6.

5. Nishimura S, Sekiya T. Human cancer and cellular oncogenes. Biochem J 1987;243:313-27.

6. Barbacid M. Ras genes. Annu Rev Biochem 1987;56: $779-827$.

7. Yamasaki H, Hollstein M, Martel N, Cabral JRP, Calendo D, Tomatis L. Transplacental induction of specific mutation in fetal Ha-ras and its critical role in post-natal carcinogenesis. Int J Cancer 1987;40:81822.

8. Klein G. The approaching era of the tumour suppressor genes. Science 1987;238:1539-45.

9. Knudson AG Jr. Hereditary cancer, oncogenes and antioncogenes. Cancer Res 1985;45:1437-43.

10. Wilson JG. Current status of teratology. In: Wilson JG, Fruser FC, ed. Handbook of teratology. New York, New York: Plenum Press, 1977:47-74.

11. Reynolds SH, Stowers SJ, Patterson RM, Maronpot RR, Aaronson RM, Anderson MW. Activated oncogenes in B6C3Fl mouse liver tumors: implications for risk assessment. Science 1987;231:1309-16.

12. Lowenstein WR. Junctional intercellular communication: the cell-to-cell membrane channel. Physiol Rev. 1981;61:829-913.

13. Trosko JE, Chang CC. Role of intercellular communication in tumor promotion. In: Slaga TJ, ed. Tumor promotion and carcinogenesis in vitro; vol IV. Boca Raton, Florida: CRC Press, 1984:119-85.

14. Trosko JE, Yotti LP, Warren ST, Tsushimoto G, Chang C. Inhibition of cell-cell communication by tumour promotors. Carcinog Compr Surv 1987;7:565-85.

15. Loch-Caruso R, Trosko JE. Inhibited intercellular communication as a mechanistic link between teratogenesis and carcinogenesis. CRC Crit Rev Toxicol 1987;16: $157-83$

16. O'Malley BW. Steroid hormone action in eucaryotic cells. J Clin Invest 1984;74:307-12. 
17. Poland A, Knutson JC. 2,3,7,8-Tetrachlorodibenzo$p$-dioxin and related halogenated aromatic hydrocarbons: examination of the mechanism of toxicity. Ann Rev Pharmacol Toxicol 1982;22:517-54.

18. Poland A. Reflections on the mechanism of action on halogenated aromatic hydrocarbons. In: Poland A, Kimbrough R, ed. Biological mechanisms of dioxin action. Cold Spring Harbor, New York: Cold Spring Harbor Press, 1985:109-17. (Banbury Report no 18.)

19. Umbreit TH, Gallo MA. Physiological implications of estrogen receptor modulation by $2,3,7,8$-tetrachlorodi- benzo-p-dioxin. Toxicol Lett 1988;42:5-14.

20. Dickson RB, Lippman ME. Role of estrogens in the malignant progression of breast cancer: new perspectives. Trends Pharmacol Sci 1986; [August] 294-6.

21. Dickson RB, Lippman ME. Estrogenic regulation of growth factor secretion in human breast carcinoma. Endocr Rev 1987;8:29-43.

Received for publication: 11 November 1988 